\title{
An inverse source problem for the heat equation and the enclosure method
}

\author{
Masaru IKEHATA \\ Department of Mathematics, Faculty of Engineering \\ Gunma University, Kiryu 376-8515, JAPAN
}

2 February 2006

\begin{abstract}
An inverse source problem for the heat equation is considered. Extraction formulae for information about the time and location when and where the unknown source of the equation firstly appeared are given from a single lateral boundary measurement. New roles of the plane progressive wave solutions or their complex versions for the backward heat equation are given.
\end{abstract}

AMS: 35R30, 80A23

KEY WORDS: inverse source problem, asymptotic solution, enclosure method, heat source, heat equation, indicator function

\section{Introduction}

Let $\Omega$ be a bounded domain of $\mathbf{R}^{n}(n=1,2,3)$ with smooth boundary. Let $T$ be an arbitrary positive number. Let $u=u(x, t)$ satisfy

$$
\left.u_{t}=\triangle u+f(x, t) \text { in } \Omega \times\right] 0, T[.
$$

In this paper we consider an inverse problem for the heat equation. The problem is Inverse Problem. Assume that there exist a non negative number $T_{0}$ less than $T$ and point $x_{0} \in \Omega$ such that $f\left(x_{0}, T_{0}\right) \neq 0$ and $f(x, t)=0$ for all $0<t<T_{0}$ and all $x \in \Omega$.

Extract $T_{0}$ and information about the set $\left\{x \in \Omega \mid f\left(x, T_{0}\right) \neq 0\right\}$ from the data $\left.u\right|_{\partial \Omega \times] 0, T[}$, $\partial u /\left.\partial \nu\right|_{\partial \Omega \times] 0, T[}$ and $u(\cdot, 0)$.

The number $T_{0}$ and the set $\left\{x \in \Omega \mid f\left(x, T_{0}\right) \neq 0\right\}$ are the time and position when and where the heat source $f(x, t)$ firstly appeared. Note that one may assume that $u(\cdot, 0)=0$ in $\Omega$ and in what follows we do so.

Some related known results should be mentioned.

Yamatani-Ohnaka [23] considered the case when the source $f(x, t)$ takes the form

$$
f(x, t)=\sum_{j=1}^{N} p_{j} \delta\left(x-x_{j}, t-t_{j}\right)
$$


where the points $x_{j} \in \Omega$, times $\left.t_{j} \in\right] 0, T\left[\right.$, strength $p_{j}(<0)$ and $N$ are all unknown. Note that, in this case $T_{0}=\min _{j}\left\{t_{j}\right\}$, formally the set $\left\{x \in \Omega \mid f\left(x, T_{0}\right) \neq 0\right\}$ coincides with $\left\{x_{j} \mid T_{0}=t_{j}\right\}$ and the source becomes inactive after the time $\max _{j} t_{j}$. They made use of special solutions of the backward heat equation $v_{t}+\Delta v=0$ in $\left.\Omega \times\right] 0, T^{\prime}[$ where $T \geq T^{\prime}>\max _{j} t_{j}$ having a large parameter $c$. Those solutions have singularity at $t=T^{\prime}$ on the given plane outside $\bar{\Omega}$ and are vanishing at $t \uparrow T^{\prime}$ on $\bar{\Omega}$. Those are constructed from the fundamental solution of the backward heat equation in one-dimensional space. Then, using integration by parts, they obtain a system of equations involving those solutions, unknown $x_{j}, t_{j}, p_{j}$ and $N$. Carefully analyzing the asymptotic behaviour of the system as $c \longrightarrow \infty$, they gave a reconstruction formula of the source itself.

Yamamoto [22] considered an inverse source problem for the wave equation, however, it is possible to apply his method to the inverse source problem for the heat equation. One interpretation of his method for the heat equation is the following. The assumption on the heat source is that

$$
f(x, t)=\sigma(t) f(x)
$$

where $\sigma$ is a known function with $\sigma(0) \neq 0$ and $f$ is unknown. From our point of view this is the case when $T_{0}$ is known and $T_{0}=0$.

Then, his method consists of two parts:

- a way of calculating the data $\left.\partial w /\left.\partial \nu\right|_{\partial \Omega} \times\right] 0, T[$ where $w$ solves

$$
\begin{gathered}
\left.w_{t}=\triangle w \text { in } \Omega \times\right] 0, T[, \\
w(x, 0)=f(x) \text { in } \Omega, \\
w(x, t)=0 \text { on } \partial \Omega \times] 0, T[,
\end{gathered}
$$

from the data $\left.\left.u\right|_{\partial \Omega} \times\right] 0, T\left[, \partial u /\left.\partial \nu\right|_{\partial \Omega} \times\right] 0, T\left[\right.$ and the time derivative $\left.(\partial / \partial t) \partial u /\left.\partial \nu\right|_{\partial \Omega} \times\right] 0, T[$;

- reconstruction formula of $f(x)$ itself from the calculated data $\left.\partial w /\left.\partial \nu\right|_{\partial \Omega} \times\right] 0, T[$. The first part can be done by solving a Volterra equation of the second kind. For establishing the second part the null-controllability for the heat equation [7, 20] and the completeness of the eigenfunctions of the Dirichlet Laplacian in $\Omega$ are essential.

In [3] El Badia-Ha Duong also considered the case when $T_{0}=0$. They assume that: the source is supported in a subset of $\Omega$ (mainly the point sources) that is independent of the time, the strength of the source on the set does not depend on the position and has a definite signature in an interval $] 0, T_{\star}\left[\right.$ with $T_{\star}<T$; the source becomes inactive after the time $T_{\star}$.

Their method also consists of two parts:

- a way of calculating the temperature in $\Omega$ at $t=T_{1}$ from the data $\left.\left.u\right|_{\partial \Omega} \times\right] T_{1}, T[$ and $\left.\partial u /\left.\partial \nu\right|_{\partial \Omega} \times\right] T_{1}, T\left[\right.$ where $T_{1}$ is an arbitrary fixed time with $T_{\star}<T_{1}<T$;

- extracting moments of the source from $u\left(x, T_{1}\right)$ and the data and determination of the sources form the moments.

The first part is also an application of the null-controllability for the heat equation and the completeness of the eigenfunctions of the Dirichlet Laplacian in $\Omega$. In the second part they start with the determination of the number and location of point sources. This is purely algebraic and can be done by using a previous result in the inverse point source problem for elliptic equation [2]. The next step is the determination of the strength of the point sources from some exponential moments which is a combination of the Müntz's theorem 
(e.g., [8]) and an invertibility of a matrix involving special solutions of the Helmholtz equations. As they pointed out the choice of the matrix is not constructive. These steps can be done by a combination of an integration by parts and special solutions for the backward heat equation.

It should be pointed out that the controllability for the heat equation plays an important role also in the problem of recovering the conductivity coefficient of the heat equation, see Avdonin-Belishev-Rozkov [1].

In this paper we apply the enclosure method to Inverse Problem which was introduced by Ikehata [13] for inverse boundary value problems for the elliptic equations. In [10] Ikehata considered in two-dimensions, an inverse source problem for the inhomogeneous Helmholtz equation $\triangle u+k^{2} u=f$ with the unknown source $f$ having the form $\chi_{D}(x) \rho(x)$ and established an extraction formula of the convex hull of $D$ provided both $D$ and $\rho$ are unknown and $D$ is polygonal. Now the enclosure method has been applied to inverse boundary value problems $[11,12,18]$, a Cauchy problem for the stationary Schrödinger equation [14] and inverse obstacle scattering problems $[15,16,17]$. The common character of these problems is: the governing equation is elliptic.

It is quite curious to consider whether one can apply the idea of the enclosure method to the case when the governing equation is non elliptic since there are many inverse problems whose governing equations are non elliptic. The inverse source problem for the heat equation is a typical and important one. The aim of this paper is to introduce a direct approach to the inverse source problem for the heat equation by employing the idea of the enclosure method.

The main feature of our method is:

- it is based on a simple one line formula;

- we do not make use of the exact controllability of the heat equation nor the completeness of the eigenfunctions of the Dirichlet Laplacian in $\Omega$;

- the assumptions on the unknown source is quite general;

- the method provides us a brief information about the time and the location when and where the unknown source firstly appeared instead of the detailed information of the source.

For several other formulations of the inverse source problem for the heat equation and results including uniqueness and stability see Cannon-Esteva [5], Cannon-DuChateau [4], Isakov [19], Hetllich-Rundell [9], recent publication of Trong-Long-Alain [21] and references therein.

\section{Statement of results}

The results of this paper are divided into two parts. The first one is a formula for extracting $T_{0}$ only and second is for extracting both $T_{0}$ and information about the location of the set $\left\{x \in \Omega \mid f\left(x, T_{0}\right) \neq 0\right\}$.

2.1. Extracting $T_{0}$.

The list of the assumptions on the source $f(x, t)$ is the following.

(A.1) $f(x, t)$ takes the form

$$
f(x, t)=\chi_{D}(x, t) \rho(x, t)
$$

where $D \subset \bar{\Omega} \times\left[T_{0}, T\right]$ is a Lebesgue measurable set; $\rho$ is essentially bounded on $D$. 
Thus the source may appear at the time $T_{0}$ firstly and the points in $D \cap\left(\mathbf{R}^{n} \times\left\{T_{0}\right\}\right)$. The next assumption describes the standing behaviour of the source.

(A.2) There exist positive numbers $\delta<T-T_{0}, C_{1}, C_{2}$ and $p \in[0, \infty[$ such that the $n$-dimensional Lebesgue measure of the set $D(s) \equiv D \cap\left(\mathbf{R}^{n} \times\left\{T_{0}+s\right\}\right)$ has the lower estimate:

$$
|D(s)| \geq C_{1} s^{p} \text { for almost all } s \in\left[0, \delta_{1}\right] ;
$$

the strength of the source $\rho$ satisfies

$$
\rho(x, t) \geq C_{2} \text { for almost all }(x, t) \in D \cap\left(\mathbf{R}^{n} \times\left[T_{0}, T_{0}+\delta\right]\right)
$$

or

$$
-\rho(x, t) \geq C_{2} \text { for almost all }(x, t) \in D \cap\left(\mathbf{R}^{n} \times\left[T_{0}, T_{0}+\delta\right]\right) .
$$

Definition 2.1. Given $\omega \in S^{n-1}, s \in \mathbf{R}$ define the indicator function $I_{\omega}(\tau ; s)$ by the formula

$$
I_{\omega}(\tau ; s)=e^{\tau s} \int_{0}^{T} \int_{\partial \Omega}\left(\frac{\partial v}{\partial \nu} u-\frac{\partial u}{\partial \nu} v\right) d S d t, \tau>0
$$

where $v=v(x, t)=e^{\sqrt{\tau} x \cdot \omega-\tau t}$.

The function $v$ takes positive values and satisfies the backward heat equation $v_{t}+\triangle v=0$ in the whole space. Moreover $e^{\tau s} v$ has the special character:

- if $t>s$, then $\lim _{\tau \longrightarrow \infty} e^{\tau s} v(x, t)=0$;

- if $t<s$, then $\lim _{\tau \longrightarrow \infty} e^{\tau s} v(x, t)=\infty$.

Note that the function $w(x)=e^{\sqrt{\tau} x \cdot \omega}$ satisfies the equation

$$
\triangle w-\tau w=0
$$

in the whole space. Since $\tau>0$, this is not the Helmholtz equation. In the following we make use of the asymptotic behaviour of $w$ as $\tau \longrightarrow \infty$.

Theorem 2.1. Assume that assumptions (A.1), (A.2) are satisfied. As $\tau \longrightarrow \infty$ we have

$$
\lim _{\tau \longrightarrow \infty} \frac{\log \left|I_{\omega}(\tau ; 0)\right|}{\tau}=-T_{0}
$$

Moreover, we have:

if $s<T_{0}$, then $\lim _{\tau \longrightarrow \infty}\left|I_{\omega}(\tau ; s)\right|=0$;

if $s>T_{0}$, then $\lim _{\tau \longrightarrow \infty}\left|I_{\omega}(\tau ; s)\right|=\infty$.

The result can be generalized to the case when the governing equation has variable coefficients. In that case there is an interesting relationship between the high frequency asymptotic solution of the corresponding wave equation [6] and that of the formal adjoint equation of the heat equation. This is clarified in Section 3.

2.2. Extracting both $T_{0}$ and information about the set $\left\{x \in \Omega \mid f\left(x, T_{0}\right) \neq 0\right\}$.

This subsection starts with the following observation. Let $n \geq 1$. Let $c \neq 0$ be an arbitrary number. Let

$$
v(x, t)=e^{-(z \cdot z) t} e^{x \cdot z}
$$


where $\tau$ satisfies $\tau>c^{-2}$ and

$$
z= \begin{cases}c \tau\left(\omega+i \sqrt{1-\frac{1}{c^{2} \tau}} \omega^{\perp}\right), & n \geq 2, \\ c \tau\left(1+i \sqrt{1-\frac{1}{c^{2} \tau}}\right), & n=1 .\end{cases}
$$

The function $v$ is a complex valued function and satisfies the backward heat equation $v_{t}+\triangle v=0$. Moreover $e^{\tau s} v$ has the special character:

- if $s<t-c x \cdot \omega$, then $\lim _{\tau \longrightarrow \infty} e^{\tau s}|v(x, t)|=0$;

- if $s>t-c x \cdot \omega$, then $\lim _{\tau \longrightarrow \infty} e^{\tau s}|v(x, t)|=\infty$.

So one can expect that: using this function, one may obtain more information about the location and shape of $D$ in the space time. We show that the method [14] is applicable in the case when $n=1,2$ (at least).

Definition 2.2. Let $n \geq 2$. Given $c>0, s \in \mathbf{R}, \omega, \omega^{\perp} \in S^{n-1}$ with $\omega \cdot \omega^{\perp}=0$ define the indicator function $I_{\omega, \omega^{\perp}, c}(\tau ; s)$ by the formula

$$
I_{\omega, \omega^{\perp}, c}(\tau ; s)=e^{\tau s} \int_{0}^{T} \int_{\partial \Omega}\left(\frac{\partial v}{\partial \nu} u-\frac{\partial u}{\partial \nu} v\right) d S d t, \tau>c^{-2}
$$

where $v$ is the function given by $(2.2)$.

Let $n=1$. Given $c \neq 0, s \in \mathbf{R}$, define the indicator function $I_{c}(\tau ; s)$ by the formula

$$
I_{c}(\tau ; s)=e^{\tau s} \int_{0}^{T} \int_{\partial \Omega}\left(\frac{\partial v}{\partial \nu} u-\frac{\partial u}{\partial \nu} v\right) d S d t, \tau>c^{-2}
$$

where $v$ is the function given by (2.2).

Let $n \geq 2$. Given $\omega \in S^{n-1}$ and $c>0$ define the unit vector $\omega(c)$ in $\mathbf{R}^{n+1}=\mathbf{R}^{n} \times \mathbf{R}$ directed into the half space $t<0$ by the formula

$$
\omega(c)=\frac{1}{\sqrt{c^{2}+1}}\left(\begin{array}{c}
c \omega \\
-1
\end{array}\right) \text {. }
$$

Then

$$
t-c x \cdot \omega=-\sqrt{c^{2}+1}\left(\begin{array}{l}
x \\
t
\end{array}\right) \cdot \omega(c) .
$$

Thus we see that:

- if $-s / \sqrt{c^{2}+1}>(x t)^{T} \cdot \omega(c)$, then $\lim _{\tau \longrightarrow \infty} e^{\tau s}|v(x, t)|=0$;

- if $-s / \sqrt{c^{2}+1}<(x t)^{T} \cdot \omega(c)$, then then $\lim _{\tau \rightarrow \infty} e^{\tau s}|v(x, t)|=\infty$;

First we consider the case when $n=2$.

We assume that the unknown source takes the form

$$
f(x, t)=\sum_{j=1}^{N} \chi_{P_{j} \times\left[T_{j}, T\right]}(x, t) \rho_{j}(x, t)
$$

where

- $P_{j} \subset \bar{\Omega}$ is given by the interior of a polygon and $T_{j}$ satisfies $0 \leq T_{j}<T$; 
- if $j \neq j^{\prime}$, then $\bar{P}_{j} \cap \bar{P}_{j^{\prime}}=\emptyset$;

- for each $j \rho_{j} \in L^{\infty}\left(P_{j} \times\right] T_{j}, T[)$ and for each vertices $p$ of the convex hull of $P_{j} \rho_{j}$ coincides with a Hölder continuous function with exponent $\left.\left.\theta_{j} \in\right] 0,1\right]$ in a neighbourhood of $\left(p, T_{j}\right)$ that does not vanish at $\left(p, T_{j}\right)$.

We set $D=\cup_{j=1}^{N}\left(P_{j} \times\right] T_{j}, T[)$.

Theorem 2.2. Let $\omega(c)$ be regular with respect to $D$. Assume that

$$
\sup _{x \in \Omega}\left(\begin{array}{c}
x \\
T
\end{array}\right) \cdot \omega(c)<h_{D}(\omega(c)) .
$$

Then the formula

$$
\lim _{\tau \longrightarrow \infty} \frac{\log \left|I_{\omega, \omega^{\perp}, c}(\tau ; 0)\right|}{\tau}=\sqrt{c^{2}+1} h_{D}(\omega(c))
$$

is valid.

Moreover, we have:

$$
\begin{aligned}
& \text { if } h_{D}(\omega(c)) \leq-s / \sqrt{c^{2}+1}, \text { then } \lim _{\tau \longrightarrow \infty}\left|I_{\omega, \omega^{\perp}, c}(\tau ; s)\right|=0 \\
& \text { if } h_{D}(\omega(c))>-s / \sqrt{c^{2}+1} \text {, then } \lim _{\tau \longrightarrow \infty}\left|I_{\omega, \omega^{\perp}, c}(\tau ; s)\right|=\infty
\end{aligned}
$$

The condition (2.3) means that the set $\Omega \times\{T\}$ is located in the half space $(x, t)^{T} \cdot \omega(c)<$ $h_{D}(\omega(c))$. This can be satisfied in the case when: $T$ is given and $c$ is sufficiently small; $c$ is given and $T$ is sufficiently large.

Next we consider the case when $n=1$.

We assume that the unknown source takes the form

$$
f(x, t)=\sum_{j=1}^{N} \chi_{P_{j} \times\left[T_{j}, T\right]}(x, t) \rho_{j}(x, t)
$$

where

- $P_{j} \subset \bar{\Omega}$ is given by the interior of an interval and $T_{j}$ satisfies $0 \leq T_{j}<T$;

- if $j \neq j^{\prime}$, then $\bar{P}_{j} \cap \bar{P}_{j^{\prime}}=\emptyset$;

- for each $j \rho_{j} \in L^{\infty}\left(P_{j} \times\right] T_{j}, T[)$ and for each end point $p$ of $P_{j} \rho_{j}$ coincides with a function of class $C^{2}$ in a neighbourhood of the point $\left(p, T_{j}\right)$ that does not vanish at $\left(p, T_{j}\right)$. We set $D=\cup_{j=1}^{N}\left(P_{j} \times\right] T_{j}, T[)$.

Given $c \neq 0$ define the unit vector $\omega(c)$ in $\mathbf{R}^{1+1}=\mathbf{R} \times \mathbf{R}$ directed into the half space $t<0$ by the formula

$$
\omega(c)=\frac{1}{\sqrt{c^{2}+1}}\left(\begin{array}{c}
c \\
-1
\end{array}\right) .
$$

Theorem 2.3. Let $\omega(c)$ be regular with respect to $D$. Assume that

$$
\sup _{x \in \Omega}\left(\begin{array}{c}
x \\
T
\end{array}\right) \cdot \omega(c)<h_{D}(\omega(c))
$$

Then the formula

$$
\lim _{\tau \longrightarrow \infty} \frac{\log \left|I_{c}(\tau ; 0)\right|}{\tau}=\sqrt{c^{2}+1} h_{D}(\omega(c))
$$

is valid.

Moreover, we have: 
if $h_{D}(\omega(c)) \leq-s / \sqrt{c^{2}+1}$, then $\lim _{\tau \longrightarrow \infty}\left|I_{c}(\tau ; s)\right|=0$;

if $h_{D}(\omega(c))>-s / \sqrt{c^{2}+1}$, then $\lim _{\tau \longrightarrow \infty}\left|I_{c}(\tau ; s)\right|=\infty$.

If $\Omega \subset \mathbf{R}^{3}$, the situation seems more complicated. We will consider the case in the future work.

\section{Proof of Theorem 2.1 and its generalization}

\subsection{Proof of Theorem 2.1.}

Since we have the trivial identity

$$
I_{\omega}(\tau ; s)=e^{\tau\left(s-T_{0}\right)} I_{\omega}\left(\tau ; T_{0}\right) \forall s,
$$

it suffices to study the asymptotic behaviour of the indicator function at $s=T_{0}$. Integration by parts gives

$$
\int_{0}^{T} \int_{\Omega} f(x, t) v(x, t) d x d t=\int_{\Omega} u(x, T) v(x, T) d x+\int_{0}^{T} \int_{\partial \Omega}\left(\frac{\partial v}{\partial \nu} u-\frac{\partial u}{\partial \nu} v\right) d S d t .
$$

Thus we have the representation of the indicator function at $s=T_{0}$ :

$$
I_{\omega}\left(\tau ; T_{0}\right)=e^{\tau T_{0}} \int_{0}^{T} \int_{\Omega} f(x, t) v(x, t) d x d t-e^{\tau T_{0}} \int_{\Omega} u(x, T) v(x, T) d x .
$$

Since $T>T_{0}$, it is easy to see that the absolute value of the second term of the right hand side is dominated by

$$
\int_{\Omega}|u(x, T)| d x e^{\sqrt{\tau} \sup _{x \in \Omega} x \cdot \omega} e^{-\tau\left(T-T_{0}\right)}=O\left(e^{-\tau\left(T-T_{0}\right) / 2}\right)
$$

as $\tau \longrightarrow \infty$.

On the other hand, from the assumptions (A.1) and (A.2) one knows that the first term of the right hand side of (3.2) takes the form

$$
\begin{gathered}
e^{\tau T_{0}} \int_{D} \rho(x, t) v(x, t) d x d t \\
=e^{\tau T_{0}} \int_{D \cap\left(\mathbf{R}^{n} \times\left[T_{0}, T_{0}+\delta\right]\right)} \rho(x, t) e^{\sqrt{\tau} x \cdot \omega-\tau t} d x d t+e^{\tau T_{0}} \int_{D \backslash\left(\mathbf{R}^{n} \times\left[T_{0}, T_{0}+\delta\right]\right)} \rho(x, t) e^{\sqrt{\tau} x \cdot \omega-\tau t} d x d t .
\end{gathered}
$$

Since the set $D \backslash\left(\mathbf{R}^{n} \times\left[T_{0}, T_{0}+\delta\right]\right.$ is contained in $\mathbf{R}^{n} \times\left[T_{0}+\delta, T\right]$, we have

$$
e^{\tau T_{0}} \int_{D \backslash\left(\mathbf{R}^{n} \times\left[T_{0}, T_{0}+\delta\right]\right.} \rho(x, t) e^{\sqrt{\tau} x \cdot \omega-\tau t} d x d t=O\left(e^{-\tau \delta / 2}\right)
$$

as $\tau \longrightarrow \infty$.

Here we prepare

Lemma 3.1. As $\tau \longrightarrow \infty$ we have

$$
K_{1} e^{\sqrt{\tau} K_{2}} \tau^{-(p+1)} \leq e^{\tau T_{0}}\left|\int_{D \cap\left(\mathbf{R}^{n} \times\left[T_{0}, T_{0}+\delta\right]\right)} \rho(x, t) e^{\sqrt{\tau} x \cdot \omega-\tau t} d x d t\right| \leq K_{3} e^{\sqrt{\tau} K_{4}}
$$

where $K_{1}, K_{2}, K_{3}, K_{4}$ are constant and $K_{1}, K_{3}>0$. 
Proof.

We just describe the key point of the proof of the left half of the inequalities in the case when

$$
\rho(x, t) \geq C_{2} \text { for almost all }(x, t) \in D \cap\left(\mathbf{R}^{n} \times\left[T_{0}, T_{0}+\delta\right]\right) .
$$

We have

$$
\begin{gathered}
e^{\tau T_{0}} \int_{D \cap\left(\mathbf{R}^{n} \times\left[T_{0}, T_{0}+\delta\right]\right)} \rho(x, t) e^{\sqrt{\tau} x \cdot \omega-\tau t} d x d t \geq C_{2} e^{\tau T_{0}} \int_{D \cap\left(\mathbf{R}^{n} \times\left[T_{0}, T_{0}+\delta\right]\right)} e^{\sqrt{\tau} x \cdot \omega-\tau t} d x d t \\
=C_{2} \int_{T_{0}}^{T_{0}+\delta} e^{-\tau\left(t-T_{0}\right)}\left(\int_{D\left(t-T_{0}\right)} e^{\sqrt{\tau} x \cdot \omega} d x\right) d t \\
=C_{2} \int_{0}^{\delta} e^{-\tau s}\left(\int_{D(s)} e^{\sqrt{\tau} x \cdot \omega} d x\right) d s \\
\geq C_{2} e^{\sqrt{\tau} \inf _{x \in \Omega} x \cdot \omega} \int_{0}^{\delta}|D(s)| e^{-\tau s} d s \\
\geq C_{1} C_{2} e^{\sqrt{\tau} \inf _{x \in \Omega} x \cdot \omega} \int_{0}^{\delta} s^{p} e^{-\tau s} d s \\
=C_{1} C_{2} \tau^{-(p+1)} e^{\sqrt{\tau} \inf _{x \in \Omega} x \cdot \omega} \int_{0}^{\tau \delta} \xi^{p} e^{-\xi} d \xi .
\end{gathered}
$$

Now Theorem 2.1 is a consequence of (3.1) to (3.6).

\subsection{A generalization}

In this subsection we give a generalization of Theorem 2.1 to the heat equation with variable coefficients:

$$
a u_{t}=\nabla \cdot \gamma \nabla u+F(x, t)
$$

where $a=a(x)$ is a smooth function with positive values and $\gamma=\gamma(x)$ is a $n \times n$-real symmetric positive definite matrix valued smooth function. We apply the idea of the enclosure method to this equation. For the purpose we construct a solution with large parameter $\tau$ of the equation

$$
a v_{t}+\nabla \cdot \gamma \nabla v=0
$$

which plays the role of the function $e^{\sqrt{\tau} x \cdot \omega-\tau t}$ in Theorem 2.1.

First we consider how to construct a solution of the equation (3.7) in the form:

$$
v(x, t)=e^{-\tau t} w(x) .
$$

Substituting this into (3.7), we have

$$
\nabla \cdot \gamma \nabla w-\tau a w=0
$$

Note that $\tau>0$ unlike the case when the governing equation is the wave equation ([6]). Let $\varphi$ be a smooth function. The change of the dependent variable

$$
w=e^{\sqrt{\tau} \varphi} w^{\prime}
$$


gives

$$
\nabla \cdot \gamma \nabla w^{\prime}+\sqrt{\tau}\left(2 \gamma \nabla \varphi \cdot \nabla w^{\prime}+(\nabla \cdot \gamma \nabla \varphi) w^{\prime}\right)+\tau((\gamma \nabla \varphi \cdot \nabla \varphi)-a) w^{\prime}=0
$$

Thus if $\varphi$ satisfies the eikonal equation

$$
\gamma \nabla \varphi \cdot \nabla \varphi=a
$$

then $w^{\prime}$ satisfies

$$
\nabla \cdot \gamma \nabla w^{\prime}+\sqrt{\tau}\left(2 \gamma \nabla \varphi \cdot \nabla w^{\prime}+(\nabla \cdot \gamma \nabla \varphi) w^{\prime}\right)=0
$$

The next lemma is crucial for the construction of the exact solution from the asymptotic solution.

Proposition 3.2. Let $\tau$ be an arbitrary positive number. Given $f \in L^{2}(\Omega)$ there exists a unique weak solution $u \in H^{1}(\Omega)$ of the elliptic problem

$$
\begin{gathered}
\nabla \cdot \gamma \nabla u+\sqrt{\tau}(2 \gamma \nabla \varphi \cdot \nabla u+(\nabla \cdot \gamma \nabla \varphi) u)=f \text { in } \Omega \\
u=0 \text { on } \partial \Omega .
\end{gathered}
$$

Moreover $u$ has the estimate

$$
\|u\|_{H^{1}(\Omega)} \leq C\|f\|_{L^{2}(\Omega)}
$$

where $C$ is a positive constant independent of $\tau$.

Proof. Using a change of dependent variable, one can easily deduce the uniqueness and existence of the solution for the corresponding fact for the equation (3.8) in $\Omega$. The positivity of $\tau$ is essential. The problem is the estimate (3.11). Since

$$
(2 \gamma \nabla \varphi \cdot \nabla u) u=\gamma \nabla \varphi \cdot \nabla\left(u^{2}\right)
$$

integration by parts gives

$$
\int_{\Omega}(2 \gamma \nabla \varphi \cdot \nabla u+(\nabla \cdot \gamma \nabla \varphi) u) u d x=0
$$

Then from the equation for $u$ we obtain

$$
\int_{\Omega} \gamma \nabla u \cdot \nabla u d x=-\int_{\Omega} f u d x
$$

A standard argument gives (3.11).

From the equation one knows that the solution $u$ in Proposition 3.2 satisfies $\nabla \cdot \gamma \nabla u \in$ $L^{2}(\Omega)$. A combination of the standard elliptic estimate for the operator $\nabla \cdot \gamma \nabla$ and (3.11) yields

$$
\|u\|_{H^{2}(\Omega)} \leq C^{\prime}(\sqrt{\tau}+1)\|f\|_{L^{2}(\Omega)} .
$$

Then the Sobolev imbedding yields

$$
\|u\|_{L^{\infty}(\Omega)} \leq C^{\prime \prime}(\sqrt{\tau}+1)\|f\|_{L^{2}(\Omega)} .
$$


Given $N \geq 1$ set

$$
w_{N}^{\prime}(x)=\sum_{j=0}^{N} \frac{a_{j}(x)}{(\sqrt{\tau})^{j}}
$$

where $a_{0}, \cdots, a_{N}$ satisfy the transport equations

$$
\begin{gathered}
2 \gamma \nabla \varphi \cdot \nabla a_{0}+(\nabla \cdot \gamma \nabla \varphi) a_{0}=0 \\
2 \gamma \nabla \varphi \cdot \nabla a_{j+1}+(\nabla \cdot \gamma \nabla \varphi) a_{j+1}=-\nabla \cdot \gamma \nabla a_{j}, j=0, \cdots, N-1 .
\end{gathered}
$$

Then we see that

$$
\nabla \cdot \gamma \nabla w_{N}^{\prime}+\sqrt{\tau}\left(2 \gamma \nabla \varphi \cdot \nabla w_{N}^{\prime}+(\nabla \cdot \gamma \nabla \varphi) w_{N}^{\prime}\right)=\frac{\nabla \cdot \gamma \nabla a_{N}(x)}{(\sqrt{\tau})^{N}}
$$

This means that $w_{N}^{\prime}$ is an asymptotic solution of the equation (3.10). Then Proposition 3.2 ensures the unique solvability of the problem

$$
\begin{gathered}
\nabla \cdot \gamma \nabla u+\sqrt{\tau}(2 \gamma \nabla \varphi \cdot \nabla u+(\nabla \cdot \gamma \nabla \varphi) u)=-\frac{\nabla \cdot \gamma \nabla a_{N}(x)}{(\sqrt{\tau})^{N}} \text { in } \Omega \\
u=0 \text { on } \partial \Omega
\end{gathered}
$$

Write $u=R_{N}$. Then the function

$$
w_{N}^{\prime \prime}=w_{N}^{\prime}+R_{N}
$$

is an exact solution of the equation (2.10) and, by virtue of the estimate (3.11), we have

$$
\left\|R_{N}\right\|_{H^{1}(\Omega)}=O\left(\frac{1}{(\sqrt{\tau})^{N}}\right) .
$$

Thus we have

$$
w_{N}^{\prime \prime}=\sum_{j=0}^{N-1} \frac{a_{j}(x)}{(\sqrt{\tau})^{j}}+O\left(\frac{1}{(\sqrt{\tau})^{N}}\right) .
$$

Note that $w_{N}^{\prime \prime}$ is the unique solution of the problem

$$
\begin{gathered}
\nabla \cdot \gamma \nabla u+\sqrt{\tau}(2 \gamma \nabla \varphi \cdot \nabla u+(\nabla \cdot \gamma \nabla \varphi) u)=0 \text { in } \Omega \\
u=w_{N}^{\prime} \text { on } \partial \Omega
\end{gathered}
$$

Summing up, we have

Theorem 3.3. Let $\varphi$ satisfy the eikonal equation (3.9). Given $N \geq 1$ let the functions $a_{0}, \cdots, a_{N}$ satisfy the transport equations (3.13). Then the unique solution of the elliptic problem

$$
\begin{aligned}
& \nabla \cdot \gamma \nabla w-\tau a w=0 \text { in } \Omega, \\
& w=e^{\sqrt{\tau} \varphi} \sum_{j=0}^{N} \frac{a_{j}}{(\sqrt{\tau})^{j}} \text { on } \partial \Omega
\end{aligned}
$$


satisfies, as $\tau \longrightarrow \infty$

$$
\left\|e^{-\sqrt{\tau} \varphi} w-\sum_{j=0}^{N-1} \frac{a_{j}}{(\sqrt{\tau})^{j}}\right\|_{H^{1}(\Omega)}=O\left(\frac{1}{(\sqrt{\tau})^{N}}\right) .
$$

Note that, from (3.12) we also have

$$
\left\|e^{-\sqrt{\tau} \varphi} w-\sum_{j=0}^{N-2} \frac{a_{j}}{(\sqrt{\tau})^{j}}\right\|_{L^{\infty}(\Omega)}=O\left(\frac{1}{(\sqrt{\tau})^{N-1}}\right)
$$

where $N \geq 2$.

Thus we have a special solution $v$ of the backward heat equation (3.7) in $\Omega \times \mathbf{R}^{+}$ having the form

$$
v(x, t)=e^{-\tau t} e^{\sqrt{\tau} \varphi}\left\{\sum_{j=0}^{N-1} \frac{a_{j}(x)}{(\sqrt{\tau})^{j}}+O\left(\frac{1}{(\sqrt{\tau})^{N}}\right)\right\}
$$

as $\tau \longrightarrow \infty$ provided $\varphi, a_{0}, \cdots, a_{N}$ are given.

Now we define another indicator function.

Definition 3.1. Given $s \in \mathbf{R}$ define the indicator function $I_{\varphi}(\tau ; s)$ by the formula

$$
I_{\varphi}(\tau ; s)=e^{\tau s} \int_{0}^{T} \int_{\partial \Omega}\left(\frac{\partial v}{\partial \nu} u-\frac{\partial u}{\partial \nu} v\right) d S d t, \tau>0
$$

where $v$ is given by (3.15).

For the description of the result we introduce the assumption instead of (A.2):

(A.2)' There exist positive numbers $\delta<T-T_{0}, C_{1}, C_{2}$ and $p \in[0, \infty[$ such that the $n$-dimensional Lebesgue measure of the set $D(s) \equiv D \cap\left(\mathbf{R}^{n} \times\left\{T_{0}+s\right\}\right)$ has the lower estimate:

$$
|D(s)| \geq C_{1} s^{p} \text { for almost all } s \in\left[0, \delta_{1}\right] ;
$$

the strength of the source $\rho$ multiplied by $a_{0}(x)$ satisfies

$$
\rho(x, t) a_{0}(x) \geq C_{2} \text { for almost all }(x, t) \in D \cap\left(\mathbf{R}^{n} \times\left[T_{0}, T_{0}+\delta\right]\right)
$$

or

$$
-\rho(x, t) a_{0}(x) \geq C_{2} \text { for almost all }(x, t) \in D \cap\left(\mathbf{R}^{n} \times\left[T_{0}, T_{0}+\delta\right]\right) .
$$

We obtain

Theorem 3.4. Assume that the assumptions (A.1), (A.2)' are satisfied. Then, as $\tau \longrightarrow$ $\infty$ we have

$$
\lim _{\tau \longrightarrow \infty} \frac{\log \left|I_{\varphi}(\tau ; 0)\right|}{\tau}=-T_{0}
$$

Moreover, we have:

if $s<T_{0}$, then $\lim _{\tau \longrightarrow \infty}\left|I_{\varphi}(\tau ; s)\right|=0$;

if $s>T_{0}$, then $\lim _{\tau \longrightarrow \infty}\left|I_{\varphi}(\tau ; s)\right|=\infty$.

Proof. (3.12) for $N=2$ gives the estimate

$$
\left\|w-a_{0}(\cdot)\right\|_{L^{\infty}(\Omega)}=O\left(\frac{1}{\sqrt{\tau}}\right) .
$$


Then, it follows from (A.2)' that there exists $\tau_{0}>0$ such that, for all $\tau \geq \tau_{0}$

$$
\rho(x, t) w(x) \geq C_{2} / 2 \text { for almost all }(x, t) \in D \cap\left(\mathbf{R}^{n} \times\left[T_{0}, T_{0}+\delta\right]\right)
$$

or

$$
-\rho(x, t) w(x) \geq C_{2} / 2 \text { for almost all }(x, t) \in D \cap\left(\mathbf{R}^{n} \times\left[T_{0}, T_{0}+\delta\right]\right) .
$$

Hereafter we take the same course as the proof of Theorem 2.1.

Example 3.1. Consider the simplest case: $a \equiv 1$ and $\gamma \equiv I_{n}$.

Given an arbitrary point $p \in \mathbf{R}^{n} \backslash \bar{\Omega}$ the function $\varphi$ given by the formula

$$
\varphi(x ; p)= \pm|x-p|, x \in \overline{\mathbf{R}}^{n} \backslash\{p\}
$$

satisfies the equation (3.9) for $a$ and $\gamma$ specified above. Since

$$
\triangle \varphi= \pm \frac{n-1}{|x-p|}
$$

the transport equation for $a_{0}$ in $(3.12)$ becomes

$$
(x-p) \cdot \nabla a_{0}+\frac{n-1}{2} a_{0}=0
$$

and the method of bicharacteristics yields

$$
a_{0}(x)=a_{0}\left(\frac{x-p}{|x-p|}+p\right)|x-p|^{-(n-1) / 2} .
$$

Thus if we specify the boundary value of $a_{0}$ on the unit sphere centered at $p$ as

$$
a_{0}(x)=1 \text { on }|x-p|=1,
$$

then we have

$$
a_{0}(x)=|x-p|^{-(n-1) / 2} .
$$

One can also obtain $a_{1}$ explicitly. The point is: the function $a_{0}$ specified above never vanishes on $\bar{\Omega}$. From Theorem 3.3 one obtains the solution $v=v_{ \pm}(x, t ; p)$ of the backward heat equation having the form

$$
v_{ \pm}(x, t ; p)=e^{-\tau t} e^{ \pm \sqrt{\tau}|x-p|}\left\{|x-p|^{-(n-1) / 2}+O\left(\frac{1}{\sqrt{\tau}}\right)\right\}
$$

as $\tau \longrightarrow \infty$.

\section{Proofs of Theorems 2.2 and 2.3}

\subsection{Asymptotic behaviour of an integral}

In this subsection, we consider the case when $n \geq 2$. we assume that $D \subset \mathbf{R}^{n+1}$ is a finite cone with a vertex at $p$ and a bottom face $Q$. More precisely, $D$ takes the form

$$
D=\cup_{0<s<\delta}\left\{p+\frac{s}{\delta}(y-p) \mid y \in Q\right\}
$$


and $Q$ is a bounded open subset of the $n$-dimensional hyper plane

$$
\left(\begin{array}{l}
x \\
t
\end{array}\right) \cdot \omega(c)=p \cdot \omega(c)-\delta
$$

where $\delta$ is a positive number.

We consider the integral of the function $v(x, t)$ given by $(2.2)$ over $D$ :

$$
I(\tau)=\int_{D} v(x, t) d x d t
$$

Lemma 4.1. The limit

$$
\lim _{\tau \longrightarrow \infty} \frac{2}{n !}(c \tau)^{n+1} \exp \left\{-\sqrt{c^{2}+1} \tau p \cdot \omega(c)\right\} \exp \left\{-i c \tau \sqrt{1-\frac{1}{c^{2} \tau}} p \cdot\left(\begin{array}{c}
\omega^{\perp} \\
0
\end{array}\right)\right\} I(\tau)
$$

exists and has the integral representation:

$$
K_{D}=2 \delta \int_{Q} \frac{d S(y)}{\left(\frac{\delta \sqrt{c^{2}+1}}{c}-i(y-p) \cdot\left(\begin{array}{c}
\omega^{\perp} \\
0
\end{array}\right)\right)^{n+1}}
$$

Proof.

Write

$$
\begin{gathered}
\exp \left\{-\sqrt{c^{2}+1} \tau p \cdot \omega(c)\right\} \exp \left\{-i c \tau \sqrt{1-\frac{1}{c^{2} \tau}} p \cdot\left(\begin{array}{c}
\omega^{\perp} \\
0
\end{array}\right)\right\} I(\tau) \\
=\int_{D} \exp \sqrt{c^{2}+1} \tau\left(\left(\begin{array}{c}
x \\
t
\end{array}\right)-p\right) \cdot \omega(c) \exp i c \tau \sqrt{1-\frac{1}{c^{2} \tau}}\left(\left(\begin{array}{c}
x \\
t
\end{array}\right)-p\right) \cdot\left(\begin{array}{c}
\omega^{\perp} \\
0
\end{array}\right) d x d t \\
=\int_{0}^{\delta} d s \int_{Q} d S(y)\left(\frac{s}{\delta}\right)^{n} e^{-\sqrt{c^{2}+1} \tau s} \exp \frac{i c \tau s}{\delta} \sqrt{1-\frac{1}{c^{2} \tau}}(y-p) \cdot\left(\begin{array}{c}
\omega^{\perp} \\
0
\end{array}\right) \\
=\delta^{-n} \tau^{-(n+1)} \int_{Q} d S(y) \int_{0}^{\tau \delta} d \xi \xi^{n} e^{-\sqrt{c^{2}+1} \xi} \exp \frac{i c \xi}{\delta} \sqrt{1-\frac{1}{c^{2} \tau}}(y-p) \cdot\left(\begin{array}{c}
\omega^{\perp} \\
0
\end{array}\right) .
\end{gathered}
$$

Then, a combination of Lebesgue's dominated convergence theorem and the formula

$$
\int_{0}^{\infty} \xi^{n} e^{-\xi} e^{i a \xi} d \xi=\frac{n !}{(1-i a)^{n+1}}, a \in \mathbf{R}
$$


gives

$$
\begin{gathered}
\tau^{n+1} \exp \left\{-\sqrt{c^{2}+1} \tau p \cdot \omega(c)\right\} \exp \left\{-i c \tau \sqrt{1-\frac{1}{c^{2} \tau}} p \cdot\left(\begin{array}{c}
\omega^{\perp} \\
0
\end{array}\right)\right\} I(\tau) \\
\longrightarrow \delta^{-n} \int_{Q} d S(y) \int_{0}^{\infty} d \xi \xi^{n} e^{-\sqrt{c^{2}+1} \xi} \exp \frac{i c \xi}{\delta}(y-p) \cdot\left(\begin{array}{c}
\omega^{\perp} \\
0
\end{array}\right) \\
=\delta^{-n}\left(\sqrt{c^{2}+1}\right)^{-(n+1)} \int_{Q} d S(y)\left\{\int_{0}^{\infty} \xi^{n} e^{-\xi} \exp \frac{i c \xi}{\delta \sqrt{c^{2}+1}}(y-p) \cdot\left(\begin{array}{c}
\omega^{\perp} \\
0
\end{array}\right) d \xi\right\} \\
=\frac{n !}{\delta^{n}\left(\sqrt{c^{2}+1}\right)^{n+1}} \int_{Q} \frac{d S(y)}{\left(1-\frac{i c}{\delta \sqrt{c^{2}+1}}(y-p) \cdot\left(\begin{array}{c}
\omega^{\perp} \\
0
\end{array}\right)\right)^{n+1}} \\
=\frac{n !}{2} c^{-(n+1)} K_{D} .
\end{gathered}
$$

\subsection{Non vanishing of $K_{D}$}

It seems difficult to check $K_{D} \neq 0$ by using (4.1) directly. In this subsection, we give an alternative expression of $K_{D}$ which yields $K_{D} \neq 0$ directly in the case when $n=2$ provided $D \subset \mathbf{R}^{2+1}$ is given by the interior of a tetrahedron with a vertex at $P$ with the bottom face $Q$ located on the plane $(x, t)^{T} \cdot \omega(c)=p \cdot \omega(c)-\delta$. The set $Q$ is given by the interior of the triangle with vertices $y_{1}, y_{2}, y_{3}$ which satisfy $y_{j} \cdot \omega(c)=p \cdot \omega(c)-\delta$.

Set

$$
\Delta=\{(\alpha, \beta) \mid \alpha, \beta \geq 0, \alpha+\beta \leq 1\} .
$$

Define the maps $\varphi_{1}, \varphi_{2}, \varphi_{3}: \Delta \longrightarrow \mathbf{R}^{2+1}$ by the formulae

$$
\begin{aligned}
& \varphi_{1}(\alpha, \beta)=p+\alpha\left(y_{1}-p\right)+\beta\left(y_{2}-p\right), \\
& \varphi_{2}(\alpha, \beta)=p+\alpha\left(y_{2}-p\right)+\beta\left(y_{3}-p\right), \\
& \varphi_{3}(\alpha, \beta)=p+\alpha\left(y_{3}-p\right)+\beta\left(y_{1}-p\right)
\end{aligned}
$$

and set

$$
\Delta_{j}=\varphi_{j}(\Delta) .
$$

Renumbering $y_{1}, y_{2}, y_{3}$ if necessary, one has the decomposition of $\partial D$ :

$$
\partial D=Q \cup \Delta_{1} \cup \Delta_{2} \cup \Delta_{3} .
$$

$Q$ and $\Delta_{j}, j=1,2,3$ satisfy $Q \cup \Delta_{j}=\emptyset$ and Int $\Delta_{j} \cap \operatorname{Int} \Delta_{j^{\prime}}=\emptyset$ if $j \neq j^{\prime}$.

Let $\nu$ be the unit outward normal vector field to $\partial D$. Since $\nu$ takes a constant vector on each $\Delta_{j}$, we denote the vector by $\nu_{j}$. For simplicity of description we identify $3+1$ with 
1 and $1-1$ with 3 . Renumbering $y_{1}, y_{2}, y_{3}$ if necessary, one may assume, in advance, that

$$
\begin{aligned}
& \left(\nu_{1} \times \nu_{3}\right) \cdot \omega(c)<0, \\
& \left(\nu_{2} \times \nu_{1}\right) \cdot \omega(c)<0, \\
& \left(\nu_{3} \times \nu_{2}\right) \cdot \omega(c)<0 .
\end{aligned}
$$

Proposition 4.2. The formula

$$
K_{D}\left(\begin{array}{c}
c\left(\omega+i \omega^{\perp}\right) \\
-1
\end{array}\right)=c^{3} \sum_{j=1}^{3} \frac{\left|\left(\nu_{j} \times \nu_{j-1}\right) \times\left(\nu_{j+1} \times \nu_{j}\right)\right|}{\left(\nu_{j} \times \nu_{j-1}\right) \cdot \vartheta\left(\nu_{j+1} \times \nu_{j}\right) \cdot \vartheta} \nu_{j},
$$

is valid where

$$
\vartheta=\left(\begin{array}{c}
c\left(\omega+i \omega^{\perp}\right) \\
-1
\end{array}\right)
$$

Proof. Let $\boldsymbol{a}$ be an arbitrary constant complex vector in four-dimensions. Since

$$
\nabla_{(x, t)} \cdot(v \boldsymbol{a})=\left\{\left(\begin{array}{c}
z \\
-\tau
\end{array}\right) \cdot \boldsymbol{a}\right\} v
$$

we have

$$
\left(\begin{array}{c}
z \\
-\tau
\end{array}\right) \cdot \boldsymbol{a} \int_{D} v d x d t=\sum_{j=1}^{3} \boldsymbol{a} \cdot \nu_{j} \int_{\Delta_{j}} v d S(y)-\boldsymbol{a} \cdot \omega(c) \int_{Q} v d S(y) .
$$

It is easy to see that, as $\tau \longrightarrow \infty$

$$
\int_{Q} v d S(y)=O\left(e^{\sqrt{c^{2}+1} \tau(p \cdot \omega(c)-\delta)}\right) .
$$

On the other hand, using a similar computation as in [14], we have

$$
\begin{gathered}
\int_{\Delta_{j}} v d S(y) \\
=\exp \left\{\sqrt{c^{2}+1} \tau p \cdot \omega(c)\right\} \exp \left\{i c \tau \sqrt{1-\frac{1}{c^{2} \tau}} p \cdot\left(\begin{array}{c}
\omega^{\perp} \\
0
\end{array}\right)\right\} \frac{\delta^{2}\left|\left(\nu_{j} \times \nu_{j-1}\right) \times\left(\nu_{j+1} \times \nu_{j}\right)\right|}{\left(\nu_{j} \times \nu_{j-1}\right) \cdot \omega(c)\left(\nu_{j+1} \times \nu_{j}\right) \cdot \omega(c)} \\
\times \int_{\Delta} \exp \left\{-\sqrt{c^{2}+1} \tau(\alpha+\beta) \delta\right\} \exp \left\{-i c \tau \sqrt{1-\frac{1}{c^{2} \tau}} \delta\left(\alpha c_{1}+\beta c_{2}\right)\right\} d \alpha d \beta
\end{gathered}
$$

where

$$
c_{1}=\frac{\left(\nu_{j} \times \nu_{j-1}\right) \cdot\left(\begin{array}{c}
\omega^{\perp} \\
0
\end{array}\right)}{\left(\nu_{j} \times \nu_{j-1}\right) \cdot \omega(c)}, c_{2}=\frac{\left(\nu_{j+1} \times \nu_{j}\right) \cdot\left(\begin{array}{c}
\omega^{\perp} \\
0
\end{array}\right)}{\left(\nu_{j+1} \times \nu_{j}\right) \cdot \omega(c)} .
$$


A combination of the change of variables and Lebesgue's dominated convergence theorem gives

$$
\begin{gathered}
(\delta \tau)^{2} \int_{\Delta} \exp \left\{-\sqrt{c^{2}+1} \tau(\alpha+\beta) \delta\right\} \exp \left\{-i c \tau \sqrt{1-\frac{1}{c^{2} \tau}} \delta\left(\alpha c_{1}+\beta c_{2}\right)\right\} d \alpha d \beta \\
=\int_{0}^{\tau \delta} d \beta \int_{0}^{\tau \delta-\beta} d \alpha \exp \left\{-\sqrt{c^{2}+1}(\alpha+\beta)\right\} \exp \left\{-i c \sqrt{1-\frac{1}{c^{2} \tau}}\left(\alpha c_{1}+\beta c_{2}\right)\right\} \\
\longrightarrow \int_{0}^{\infty} d \beta \int_{0}^{\infty} d \alpha \exp \left\{-\sqrt{c^{2}+1}(\alpha+\beta)\right\} \exp \left\{-i c\left(\alpha c_{1}+\beta c_{2}\right)\right\} \\
\left.=\left(c^{2}+1\right)^{-1} \int_{0}^{\infty} d \beta \int_{0}^{\infty}+1\right)^{-1} \frac{c \exp \{-(\alpha+\beta)\} \exp \left\{-i \frac{c}{\sqrt{c^{2}+1}}\left(\alpha c_{1}+\beta c_{2}\right)\right\}}{\left(1+i \frac{c}{\sqrt{c^{2}+1}} c_{1}\right)\left(1+i \frac{c}{\sqrt{c^{2}+1}} c_{2}\right)} \\
=\frac{\left(\nu_{j} \times \nu_{j-1}\right) \cdot \omega(c)\left(\nu_{j+1} \times \nu_{j}\right) \cdot \omega(c)}{\left(\nu_{j} \times \nu_{j-1}\right) \cdot \vartheta\left(\nu_{j+1} \times \nu_{j}\right) \cdot \vartheta} .
\end{gathered}
$$

A combination of (4.5) and (4.6) gives

$$
\begin{gathered}
\tau^{2} \exp \left\{-\sqrt{c^{2}+1} \tau p \cdot \omega(c)\right\} \exp \left\{-i c \tau \sqrt{1-\frac{1}{c^{2} \tau}} p \cdot\left(\begin{array}{c}
\omega^{\perp} \\
0
\end{array}\right)\right\} \int_{\Delta_{j}} v d S(y) \\
\longrightarrow \frac{\left|\left(\nu_{j} \times \nu_{j-1}\right) \times\left(\nu_{j+1} \times \nu_{j}\right)\right|}{\left(\nu_{j} \times \nu_{j-1}\right) \cdot \vartheta\left(\nu_{j+1} \times \nu_{j}\right) \cdot \vartheta}
\end{gathered}
$$

Then from this, (4.3) and (4.4) we have

$$
\begin{gathered}
\tau^{3}\left(\begin{array}{c}
c\left(\omega+i \sqrt{1-\frac{1}{c^{2} \tau}} \omega^{\perp}\right) \\
-1
\end{array}\right) \cdot \boldsymbol{a} \\
\times \exp \left\{-\sqrt{c^{2}+1} \tau p \cdot \omega(c)\right\} \exp \left\{-i c \tau \sqrt{1-\frac{1}{c^{2} \tau}} p \cdot\left(\begin{array}{c}
\omega^{\perp} \\
0
\end{array}\right)\right\} I(\tau) \\
\longrightarrow \sum_{j=1}^{3} \frac{\left|\left(\nu_{j} \times \nu_{j-1}\right) \times\left(\nu_{j+1} \times \nu_{j}\right)\right| \nu_{j} \cdot \boldsymbol{a}}{\left(\nu_{j} \times \nu_{j-1}\right) \cdot \vartheta\left(\nu_{j+1} \times \nu_{j}\right) \cdot \vartheta} .
\end{gathered}
$$


A combination of Lemma 4.1 and (4.7) yields

$$
\begin{gathered}
K_{D}\left(\begin{array}{c}
c\left(\omega+i \omega^{\perp}\right) \\
-1
\end{array}\right) \cdot \boldsymbol{a} \\
=\lim _{\tau \longrightarrow \infty}\left(\begin{array}{c}
c\left(\omega+i \sqrt{1-\frac{1}{c^{2}}} \omega^{\perp}\right) \\
-1
\end{array}\right) \cdot \boldsymbol{a} \\
\times \frac{2}{2 !}(c \tau)^{3} \exp \left\{-\sqrt{c^{2}+1} \tau p \cdot \omega(c)\right\} \exp \left\{-i c \sqrt{1-\frac{1}{c^{2} \tau}} \tau p \cdot\left(\begin{array}{c}
\omega^{\perp} \\
0
\end{array}\right)\right\} I(\tau) \\
=c^{3} \sum_{j=1}^{3} \frac{\left|\left(\nu_{j} \times \nu_{j-1}\right) \times\left(\nu_{j+1} \times \nu_{j}\right)\right| \nu_{j} \cdot \boldsymbol{a}}{\left(\nu_{j} \times \nu_{j-1}\right) \cdot \vartheta\left(\nu_{j+1} \times \nu_{j}\right) \cdot \vartheta} .
\end{gathered}
$$

Since $\boldsymbol{a}$ is arbitrary, we obtain (4.2).

Since $\nu_{1}, \nu_{2}, \nu_{3}$ are linearly independent, one has the conclusion

Corollary 4.3. $K_{D} \neq 0$ for all $c>0$.

\subsection{Proof of Theorem 2.2}

Integration by parts gives

$$
I_{\omega, \omega^{\perp}, c}(\tau ; 0)=\int_{0}^{T} \int_{\Omega} f(x, t) v(x, t) d x d t-\int_{\Omega} u(x, T) v(x, T) d x .
$$

Then (4.8) yields that the integral

$$
e^{-\tau \sqrt{c^{2}+1} h_{D}(\omega(c))} \int_{\Omega} u(x, T) v(x, T) d x
$$

is exponentially decaying as $\tau \longrightarrow \infty$. Thus the integral $e^{-\tau \sqrt{c^{2}+1} h_{D}(\omega(c))} I_{\omega, \omega^{\perp}, c}(\tau ; 0)$ modulo exponentially decaying as $\tau \longrightarrow \infty$, coincides with the integral

$$
\begin{gathered}
e^{-\tau \sqrt{c^{2}+1} h_{D}(\omega(c))} \int_{0}^{T} \int_{\Omega} f(x, t) v(x, t) d x \\
=e^{-\tau \sqrt{c^{2}+1} h_{D}(\omega(c))} \sum_{j=1}^{N} \int_{\left.P_{j} \times\right] T_{j}, T[} \rho_{j}(x, t) e^{-\tau t} e^{x \cdot z} d x d t .
\end{gathered}
$$

Since $\omega(c)$ is regular with respect to $D$, there exits a unique point $p$ on $\partial D$ such that $p \cdot \omega(c)=h_{D}(\omega(c))$. This $p$ should belong to some $P_{j_{0}} \times\left\{T_{j_{0}}\right\}$ since the time component of $\omega(c)$ is negative. Then $\left.P_{j^{\prime}} \times\right] T_{j^{\prime}}, T\left[\right.$ with $j^{\prime} \neq j_{0}$ should be contained in the half space $(x, t)^{T} \cdot \omega(c)<h_{D}(\omega)-\delta$ with a positive number $\delta$ independent of $j^{\prime}$. Thus the right hand side of (4.9) modulo exponentially decaying as $\tau \longrightarrow \infty$, coincides with

$$
e^{-\tau \sqrt{c^{2}+1} h_{D}(\omega(c))} \int_{D^{\prime}} \rho_{j_{0}}(x, t) e^{-\tau t} e^{x \cdot z} d x d t
$$

where

$$
D^{\prime}=\left(P_{j_{0}} \times\right] T_{j}, T[) \cap\left\{(x, t) \mid(x, t)^{T} \cdot \omega \geq h_{D}(\omega)-\delta\right\} .
$$


Choosing a smaller $\delta$ if necessary, one may assume that this $D^{\prime}$ is given by the interior of a tetrahedron with vertex $p$ and has the factorization as that of $D$ in subsection 4.1:

$$
D^{\prime}=\cup_{0<s<\delta}\left\{p+\frac{s}{\delta}(y-p) \mid y \in Q\right\}
$$

where $Q$ is the interior of a triangle lying on the plane $(x, t)^{T} \cdot \omega(c)=h_{D}(\omega)-\delta$. This is the key point of the proof. Divide (4.10) into two parts:

$$
\begin{gathered}
e^{-\tau \sqrt{c^{2}+1} h_{D}(\omega(c))} \rho_{j_{0}}(p) \int_{D^{\prime}} e^{-\tau t} e^{x \cdot z} d x d t+e^{-\tau \sqrt{c^{2}+1} h_{D}(\omega(c))} \int_{D^{\prime}}\left\{\rho_{j_{0}}(x, t)-\rho_{j_{0}}(p)\right\} e^{-\tau t} e^{x \cdot z} d x d t \\
\equiv I+I I .
\end{gathered}
$$

From a combination of Lemma 4.1 and Corollary 4.3 one knows that $I$ as $\tau \longrightarrow \infty$ decays really algebraically:

$$
\lim _{\tau \longrightarrow \infty}(c \tau)^{3}|I|=\left|K_{D^{\prime}}\right|\left|\rho_{j_{0}}(p)\right| .
$$

Using the Hölder continuity of $\rho_{j_{0}}$, one can also show easily

$$
\tau^{3}|I I|=O\left(\tau^{-\theta_{j_{0}}}\right)
$$

Summing up, one concludes that, as $\longrightarrow \infty$

$$
(c \tau)^{3} e^{-\tau \sqrt{c^{2}+1} h_{D}(\omega(c))}\left|I_{\omega, \omega^{\perp}, c}(\tau ; 0)\right| \longrightarrow\left|K_{D^{\prime}}\right|\left|\rho_{j_{0}}(p)\right| .
$$

Then all of the statements follow from this asymptotic formula. This completes the proof of Theorem 2.2.

\subsection{Proof of Theorem 2.3}

This is the case when $n=1$. Since

$$
z \cdot z=\tau+i 2 c^{2} \tau^{2} \sqrt{1-\frac{1}{c^{2} \tau}}
$$

we have

$$
v(x, t)=\exp \left\{\sqrt{c^{2}+1} \tau\left(\begin{array}{l}
x \\
t
\end{array}\right) \cdot \omega(c)\right\} \exp \left\{i c \tau\left(\begin{array}{c}
x \\
t
\end{array}\right) \cdot\left(\begin{array}{c}
1 \\
-2 c \tau
\end{array}\right) \sqrt{1-\frac{1}{c^{2} \tau}}\right\} .
$$

Note that this function is oscillatory higher than the case when $n \geq 2$ because of the existence of the growing factor $\tau^{2}$ in the imaginary part of the phase function.

As same as the proof of Theorem 2.2 it suffices to show that the integral

$$
\exp \left\{-\sqrt{c^{2}+1} \tau h_{D}(\omega(c))\right\} \int_{D} v(x, t) \rho(x, t) d x d t
$$

is really algebraic decaying as $\tau \longrightarrow \infty$.

Remarks are in order.

- One may assume that $D$ is given by the interior of a triangle with vertices $p, y_{0}$ and $y_{1}$ with $p \cdot \omega=h_{D}(\omega(c)), y_{0} \cdot \omega(c)=y_{1} \cdot \omega(c)=h_{D}(\omega(c))-\delta$ and has the form

$$
D=\cup_{0<s<\delta}\left\{p+\frac{s}{\delta}(y-p) \mid y \in Q\right\}
$$


where $Q$ is the segment with endpoints $y_{0}, y_{1}$. And also one may assume that $\rho_{j}, j=$ $, \cdots, N$ are given by a single $\rho \equiv \rho_{j_{0}}$ for some $j_{0}$.

- One may assume that the vectors $y_{1}-p$ and $y_{0}-p$ coincide with $\left|y_{1}-p\right|\left(\begin{array}{ll}0 & 1\end{array}\right)^{T}$ and $\left|y_{0}-p\right|(-\operatorname{sgn} c 0)^{T}$, respectively.

- Since we have assumed that $\rho$ coincides with a function of class $C^{2}$ in a neighbourhood of the point $p$ in $\mathbf{R}^{1+1}$, one may assume that, for all $(x, t) \in D$

$$
\rho(x, t)=\rho(p)+\nabla \rho(p) \cdot\left\{(x t)^{T}-p\right\}+O\left(\left|(x t)^{T}-p\right|^{2}\right)
$$

where $\rho(p)(\neq 0)$ and $\nabla \rho(p)$ are the corresponding values of the $C^{2}$-extension of the original $\rho$.

Then one has the expression

$$
\begin{gathered}
\exp \left\{-\sqrt{c^{2}+1} \tau p \cdot \omega(c)\right\} \exp \left\{-i c \tau p \cdot\left(\begin{array}{c}
1 \\
-2 c \tau
\end{array}\right) \sqrt{1-\frac{1}{c^{2} \tau}}\right\} \int_{D} v(x, t) \rho(x, t) d x d t \\
=I(\tau)+I I(\tau)+I I I(\tau)
\end{gathered}
$$

where

$$
\begin{gathered}
\frac{I(\tau)}{\rho(p)} \\
=\int_{D} \exp \left\{\sqrt{c^{2}+1} \tau\left\{\left(\begin{array}{c}
x \\
t
\end{array}\right)-p\right\} \cdot \omega(c)\right\} \exp \left\{i c \tau\left\{\left(\begin{array}{c}
x \\
t
\end{array}\right)-p\right\} \cdot\left(\begin{array}{c}
1 \\
-2 c \tau
\end{array}\right) \sqrt{1-\frac{1}{c^{2} \tau}}\right\} d x d t, \\
I I(\tau) \\
=\int_{D} \exp \left\{\sqrt{c^{2}+1} \tau\left\{\left(\begin{array}{c}
x \\
t
\end{array}\right)-p\right\} \cdot \omega(c)\right\} \exp \left\{i c \tau\left\{\left(\begin{array}{c}
x \\
t
\end{array}\right)-p\right\} \cdot\left(\begin{array}{c}
1 \\
-2 c \tau
\end{array}\right) \sqrt{1-\frac{1}{c^{2} \tau}}\right\} \\
\times \nabla \rho(p) \cdot\left\{(x t)^{T}-p\right\} d x d t,
\end{gathered}
$$

and

$$
\begin{gathered}
\operatorname{III}(\tau) \\
=\int_{D} \exp \left\{\sqrt{c^{2}+1} \tau\left\{\left(\begin{array}{c}
x \\
t
\end{array}\right)-p\right\} \cdot \omega(c)\right\} \exp \left\{i c \tau\left\{\left(\begin{array}{c}
x \\
t
\end{array}\right)-p\right\} \cdot\left(\begin{array}{c}
1 \\
-2 c \tau
\end{array}\right) \sqrt{1-\frac{1}{c^{2} \tau}}\right\} \\
\times O\left(\left|(x t)^{T}-p\right|^{2}\right) d x d t .
\end{gathered}
$$

Here we prove that, as $\tau \longrightarrow \infty I(\tau) \sim C \tau^{-3}$ with $C \neq 0$ and $I I(\tau)=I I I(\tau)=O\left(\tau^{-4}\right)$.

First we study the asymptotic behaviour of $I(\tau)$ as $\tau \longrightarrow \infty$.

$$
\begin{gathered}
I(\tau) / \rho(p)=\int_{0}^{\delta} d s \int_{Q}\left(\frac{s}{\delta}\right) e^{-s \sqrt{c^{2}+1} \tau} \exp \left\{i c \tau \frac{s}{\delta}(y-p) \cdot\left(\begin{array}{c}
1 \\
-2 c \tau
\end{array}\right) \sqrt{1-\frac{1}{c^{2} \tau}}\right\} d S(y) \\
=\frac{1}{\delta \tau^{2}} \int_{Q} d S(y) \int_{0}^{\delta \tau} \xi e^{-\xi \sqrt{c^{2}+1}} \exp \left\{i \frac{c}{\delta} \xi(y-p) \cdot\left(\begin{array}{c}
1 \\
-2 c \tau
\end{array}\right) \sqrt{1-\frac{1}{c^{2} \tau}}\right\} d \xi
\end{gathered}
$$


Set

$$
B(y, \tau)=\frac{c}{\delta}(y-p) \cdot\left(\begin{array}{c}
1 \\
-2 c \tau
\end{array}\right) \sqrt{1-\frac{1}{c^{2} \tau}}
$$

Since

$$
\begin{aligned}
& \int_{0}^{\delta \tau} \xi e^{-\xi \sqrt{c^{2}+1}} \exp \left\{i \frac{c}{\delta} \xi(y-p) \cdot\left(\begin{array}{c}
1 \\
-2 c \tau
\end{array}\right) \sqrt{1-\frac{1}{c^{2} \tau}}\right\} d \xi \\
& =\frac{1}{\left(\sqrt{c^{2}+1}-i B(y, \tau)\right)^{2}}+O\left(e^{-\tau \delta \sqrt{c^{2}+1}}\right),
\end{aligned}
$$

we obtain

$$
\tau^{2} \delta I(\tau) / \rho(p)=\int_{Q} \frac{d S(y)}{\left(\sqrt{c^{2}+1}-i B(y, \tau)\right)^{2}}+O\left(e^{-\tau \delta \sqrt{c^{2}+1}}\right) .
$$

Using a parameterization of the segment $Q$, we have

Lemma 4.4. The formulae

$$
\int_{Q} \frac{d S(y)}{\left(\sqrt{c^{2}+1}-i B(y, \tau)\right)^{2}}=\frac{\left|y_{1}-y_{0}\right|}{\left(\sqrt{c^{2}+1}-i B\left(y_{1}, \tau\right)\right)\left(\sqrt{c^{2}+1}-i B\left(y_{0}, \tau\right)\right)}
$$

and

$$
\begin{aligned}
& \int_{Q} \frac{2 \nabla \rho(p) \cdot(y-p)}{\left(\sqrt{c^{2}+1}-i B(y, \tau)\right)^{3}} d S(y)=-i \frac{\left|y_{1}-y_{0}\right|}{B\left(y_{1}, \tau\right)-B\left(y_{0}, \tau\right)} \\
& \times\left(\frac{\nabla \rho(p) \cdot\left(y_{1}-p\right)}{\left(\sqrt{c^{2}+1}-i B\left(y_{1}, \tau\right)\right)^{2}}-\frac{\nabla \rho(p) \cdot\left(y_{0}-p\right)}{\left(\sqrt{c^{2}+1}-i B\left(y_{0}, \tau\right)\right)^{2}}\right. \\
& \left.-\frac{\nabla \rho(p) \cdot\left(y_{1}-y_{0}\right)}{\left(\sqrt{c^{2}+1}-i B\left(y_{1}, \tau\right)\right)\left(\sqrt{c^{2}+1}-i B\left(y_{0}, \tau\right)\right)}\right),
\end{aligned}
$$

are valid.

Proof. Let $y=y(\eta)$ be the parameterization of $Q$ with $y(0)=y_{0}, y(1)=y_{1}$ and $y^{\prime}(\eta)=y_{1}-y_{0}$. Since

$$
\frac{d}{d \eta} B(y, \tau)=B\left(y_{1}, \tau\right)-B\left(y_{0}, \tau\right)
$$

we have

$$
\frac{d}{d \eta}\left(\frac{1}{\sqrt{c^{2}+1}-i B(y, \tau)}\right)=i \frac{B\left(y_{1}, \tau\right)-B\left(y_{2}, \tau\right)}{\left(\sqrt{c^{2}+1}-i B(y, \tau)\right)^{2}}
$$

This gives (4.12) and a combination of a similar identity and integration by parts yields also (4.13).

Since

$$
B\left(y_{1}, \tau\right)=-\frac{2 c^{2}\left|y_{1}-p\right|}{\delta} \tau \sqrt{1-\frac{1}{c^{2} \tau}}
$$


and

$$
B\left(y_{0}, \tau\right)=-\frac{|c|\left|y_{0}-p\right|}{\delta} \sqrt{1-\frac{1}{c^{2} \tau}}
$$

from (4.12) we have, as $\tau \longrightarrow \infty$

$$
\tau \int_{Q} \frac{d S(y)}{\left(\sqrt{c^{2}+1}-i B(y, \tau)\right)^{2}} \longrightarrow-i \frac{\delta}{2 c^{2}} \frac{\left|y_{1}-y_{0}\right|^{2}}{\left|y_{1}-p\right|\left(\sqrt{c^{2}+1}+i \frac{|c|}{\delta}\left|y_{0}-p\right|\right)}
$$

A combination of this and (4.11) gives the formula:

$$
\lim _{\tau \longrightarrow \infty} 2(c \tau)^{2} \tau I(\tau)=-\frac{i\left|y_{1}-y_{0}\right|^{2} \rho(p)}{\left|y_{1}-p\right|\left(\sqrt{c^{2}+1}+i \frac{|c|}{\delta}\left|y_{0}-p\right|\right)}(\neq 0) .
$$

For the estimation of $I I(\tau)$ one has to make use of the growing factor $\tau^{2}$ in the imaginary part of the phase function in the integrand. For the purpose write

$$
I I(\tau)=\frac{1}{\delta^{2} \tau^{3}} \int_{Q} \nabla \rho(p) \cdot(y-p) d S(y) \int_{0}^{\tau \delta} \xi^{2} e^{-\xi \sqrt{c^{2}+1}} e^{i \xi B(y, \tau)} d \xi .
$$

Since

$$
\int_{0}^{\tau \delta} \xi^{2} e^{-\xi \sqrt{c^{2}+1}} e^{i \xi B(y, \tau)} d \xi=\frac{2}{\left(\sqrt{c^{2}+1}-i B(y, \tau)\right)^{3}}+O\left((\tau \delta)^{2} e^{-\tau \delta \sqrt{c^{2}+1}}\right)
$$

we have

$$
\delta^{2} \tau^{3} I I(\tau)=\int_{Q} \frac{2 \nabla \rho(p) \cdot(y-p) d S(y)}{\left(\sqrt{c^{2}+1}-i B(y, \tau)\right)^{3}}+O\left((\tau \delta)^{2} e^{-\tau \delta \sqrt{c^{2}+1}}\right) .
$$

Here, from (4.13) we have, as $\tau \longrightarrow \infty$

$$
\tau \int_{Q} \frac{2 \nabla \rho(p) \cdot(y-p) d S(y)}{\left(\sqrt{c^{2}+1}-i B(y, \tau)\right)^{3}} \longrightarrow-\frac{i \delta}{2 c^{2}} \frac{\left|y_{1}-y_{0}\right|}{\left|y_{1}-p\right|} \frac{\nabla \rho(p) \cdot\left(y_{0}-p\right)}{\left(\sqrt{c^{2}+1}+i \frac{|c|}{\delta}\left|y_{0}-p\right|\right)^{2}}
$$

This gives the estimate $I I(\tau)=O\left(\tau^{-4}\right)$ as $\tau \longrightarrow \infty$. The estimation of $I I I(\tau)$ is rather easier than that of $I I(\tau)$ :

$$
\begin{aligned}
& |I I I(\tau)| \leq C \int_{0}^{\delta} d s \int_{Q}\left(\frac{s}{\delta}\right) e^{-s \tau \sqrt{c^{2}+1}}\left(\frac{s}{\delta}\right)^{2}|y-p|^{2} d S(y) \\
& \leq \frac{C}{\tau^{4}} \int_{0}^{\infty}\left(\frac{\xi}{\delta}\right)^{3} e^{-\xi \sqrt{c^{2}+1}} d \xi \int_{Q}|y-p|^{2} d S(y)=O\left(\tau^{-4}\right) .
\end{aligned}
$$

Summing up, we conclude the existence of the nonzero limit of the integral

$$
\tau^{3} \exp \left\{-\sqrt{c^{2}+1} \tau p \cdot \omega(c)\right\} \exp \left\{-i c \tau p \cdot\left(\begin{array}{c}
1 \\
-2 c \tau
\end{array}\right) \sqrt{1-\frac{1}{c^{2} \tau}}\right\} \int_{D} v(x, t) \rho(x, t) d x d t
$$


as $\tau \longrightarrow \infty$. This completes the proof of Theorem 2.3.

\section{Acknowledgement}

This research was partially supported by Grant-in-Aid for Scientific Research (C)(2) (No. 15540154) of Japan Society for the Promotion of Science.

\section{References}

[1] Avdonin, S. A., Belishev, M. I. and Rozhkov, Yu. S., The BC-method in the inverse problem for the heat equation, J. Inv. Ill-Posed Problems, 5(1997), 309-322.

[2] EL Badia, A. and Ha Duong, T., An inverse source problem in potential analysis, Inverse Problems, 16(2000), 651-663.

[3] EL Badia, A. and Ha Duong, T., On an inverse source problem for the heat eqution. Application to a pollution detection problem, J. Inv. Ill-Posed Problems, 10(2002), 585-599.

[4] Cannon, J. R. and DuChateau, P., Structual identification of an unknown source term in a heat equation, Inverse Problems, 14(1998), 535-551.

[5] Cannon, J. R. and Esteva, S. P., An inverse problem for the heat equation, Inverse Problems, 2(1986), 395-403.

[6] Egorov, V., Yu. and Shubin, M. A., I. Linear Partial Differential Equations. Elements of the Modern Theory, Partial Differential Equations II, Egorov, V. Yu. and Subin, M. A. (Eds.), Encyclopaedia of Mathematical Sciences, 31, 82-86, Springer, 1988.

[7] Fursikov and Imanuvilov, Contorollability of Evolution Equations, Lecture Notes Series 34. RIM-GARC, Seoul National University, 1996.

[8] Gurariy, V. I. and Lusky, W., Geometry of Müntz spaces and related questions, Lecture Notes in Math., 1870, Springer, 2005.

[9] Hettlich, F. and Rundell, W., Identification of a discontinuous source in the heat equation, Inverse Problems, 17(2001), 1465-1482.

[10] Ikehata, M., Reconstruction of a source domain from the Cauchy data, Inverse Problems, 15(1999), 637-645.

[11] Ikehata, M., Enclosing a polygonal cavity in a two-dimensional bounded domain from Cauchy data, Inverse Problems, 15(1999), 1231-1241.

[12] Ikehata, M., On reconstruction in the inverse conductiviy problem with one measurement, Inverse Problems, 16(2000), 785-793. 
[13] Ikehata, M., Reconstruction of the support function for inclusion from boundary measurements, J. Inv. Ill-Posed Problems, 8(2000), 367-378.

[14] Ikehata, M., Exponentially growing solutions and the Cauchy problem, Appl. Anal., 78(2001), no. 1-2, 79-95.

[15] Ikehata, M., Inverse scattering problems and the enclosure method, Inverse Problems, 20(2004), 533-551.

[16] Ikehata, M., An inverse transmission scattering problem and the enclosure method, Computing, 75(2005), 133-156.

[17] Ikehata, M., The Herglotz wave function, the Vekua transform and the enclosure method, Hiroshima Math. J., 35(2005), 485-506.

[18] Ikehata, M. and Ohe, T., A numerical method for finding the convex hull of polygonal cavities using the enclosure method, Inverse Problems, 18(2002), 111-124.

[19] Isakov, V., Inverse source problems, Mathematical surveys and monographs, No. 34, Americal Mathematical Society, Providence, Rhode Island, 1990.

[20] Lebeau, G and Robbiano, L., Contrôle exact de l'équation de la chaleur, Comm. PDE, 20(1995), 335-356.

[21] Trong, D. D., Long, T. N. and Alain, D. N. P., Nonhomogeneous heat equation: Identification and regularization for the inhomogeneous term, J. Math. Anal. Appl., 312(2005), 93-104.

[22] Yamamoto, M., Stability, reconstruction formula and regularization for an inverse source hyperbolic problem by a control method, Inverse Problems, 11(1995), 481-496.

[23] Yamatani, K. and Ohnaka, K., An estimation method for point sources of multidimensional diffusion equation, Appl. Math. Modelling, 21(1997), 77-84.

e-mail address

ikehata@math.sci.gunma-u.ac.jp 